\title{
Gluon propagator without lattice Gribov copies on a finer lattice
}

\author{
C. Alexandrou \\ Department of Physics, University of Cyprus, P.O. Box 20537, CY-1678 Nicosia, Cyprus \\ Ph. de Forcrand \\ Institut für Theoretische Physik, ETH Hönggerberg, CH-8093 Zürich, Switzerland \\ and CERN, Theory Division, CH-1211 Geneva 23, Switzerland \\ E. Follana \\ Department of Physics, University of Cyprus, P.O. Box 20537, CY-1678 Nicosia, Cyprus
}

(Received 21 December 2001; published 25 June 2002)

\begin{abstract}
We extend our study of the gluon propagator in quenched lattice QCD using the Laplacian gauge to a finer lattice. We verify the existence of a pole mass as we take the continuum limit and deduce a value of $\sim 600_{-30}^{+150} \mathrm{MeV}$ for this pole mass. We find a finite value of $[454(5) \mathrm{MeV}]^{-2}$ for the renormalized zeromomentum propagator, in agreement with results on coarser lattices.
\end{abstract}

DOI: 10.1103/PhysRevD.65.114508

PACS number(s): 11.15.Ha, 12.38.Aw, 12.38.Gc, 14.70.Dj

\section{INTRODUCTION}

The gluon propagator, although not an observable quantity, plays an important role in phenomenological nonperturbative studies. A framework for such studies is provided, for instance, by the Dyson-Schwinger equations (DSE) which recently have been applied, among other topics, to the study of the quark gluon plasma [1]. This application is particularly important because it complements experimental activity at the BNL Relativistic Heavy Ion Collider (RHIC) and also because of the difficulties of applying lattice QCD to the case of a nonzero chemical potential, although some progress is being achieved in this direction [2]. However in the Dyson-Schwinger approach the study of the gluon propagator is still inconclusive because of the various truncations needed to solve the coupled set of equations [1]. For instance, in the ghost-free axial gauge, many studies which used a simplified version of the three-gluon vertex supported an infrared-enhanced gluon propagator of the form $1 / q^{4}$. Such behavior, driven by the vacuum polarization diagram, was disputed by other DSE studies [3] as being due to a flaw in setting to zero the second scalar function that enters in the definition of the gluon propagator. Similar disputes also occur in the case of the Landau gauge where some studies, which assume dominance of the gluon-vacuum polarization, find infrared enhancement [4] whereas others [5], which use a rational polynomial ansatz for the self-energies and vertices, find an infrared vanishing propagator. After including the ghost propagator, recent studies in the Landau gauge favor an infrared-vanishing gluon propagator [6].

Lattice QCD provides a well defined theoretical framework for nonperturbative physics and it is well suited for the study of the gluon propagator. A series of papers [7], which have appeared over the past couple of years, provide a detailed study of the behavior of the gluon propagator in quenched lattice QCD in the Landau gauge. However, fixing to Landau gauge on the lattice is an iterative procedure which stops upon reaching any of a large number of local minima, called "Gribov copies". Since the global minimum itself cannot be reached, the effect of trading it for a local minimum is largely unknown. This uniqueness of the gauge condition puts us in a good position to obtain physical results on quantities, such as the pole mass which one may expect to be gauge invariant, and compare to the corresponding values used in phenomenology. The issue of gauge invariance of the gluon pole mass was addressed in Ref. [9]. For a certain class of covariant gauges, the Ward identities that determine the gauge dependence of QCD dispersion relations were derived. Using these relations one can examine the gauge independence of the poles of the gluon propagator. It was argued that if the loop expansion holds, then a nonzero pole of the transverse gluon propagator is gauge invariant.

In Ref. [8], we showed that a good description of the gluon propagator was provided by an ansatz which admits a dynamically generated gluon mass [10] and thus points to an infrared regularized gluon propagator. By analytic continuation to negative values of $q^{2}$ we obtained an estimate of the pole mass. The existence of a gluon mass has important phenomenological implications [11]. Total cross sections in hadron-hadron collisions, proton-proton elastic scattering and diffractive phenomena can be well understood if there is a finite correlation length for the gluon field [12]. For instance in the Pomeron exchange model of Landshoff and Nachtmann [13] a gluon propagator which is infrared finite is shown to eliminate the troublesome singularity in the Pomeron calculation of hadron-hadron scattering. Whereas a bare gluon mass would lead to problems with unitarity, a dynamically generated mass vanishing in the ultraviolet reproduces the correct perturbative result for the gluon propagator and is consistent with unitarity.

It is the purpose of the present work to check the robustness of our earlier results on the gluon propagator as we take the continuum limit. We thus extend our previous calculation on coarser lattices [8] to a finer lattice at $\beta=6.2$. In Ref. [8] we included a comparison of results in different physical volumes demonstrating that, for the quantities of interest 


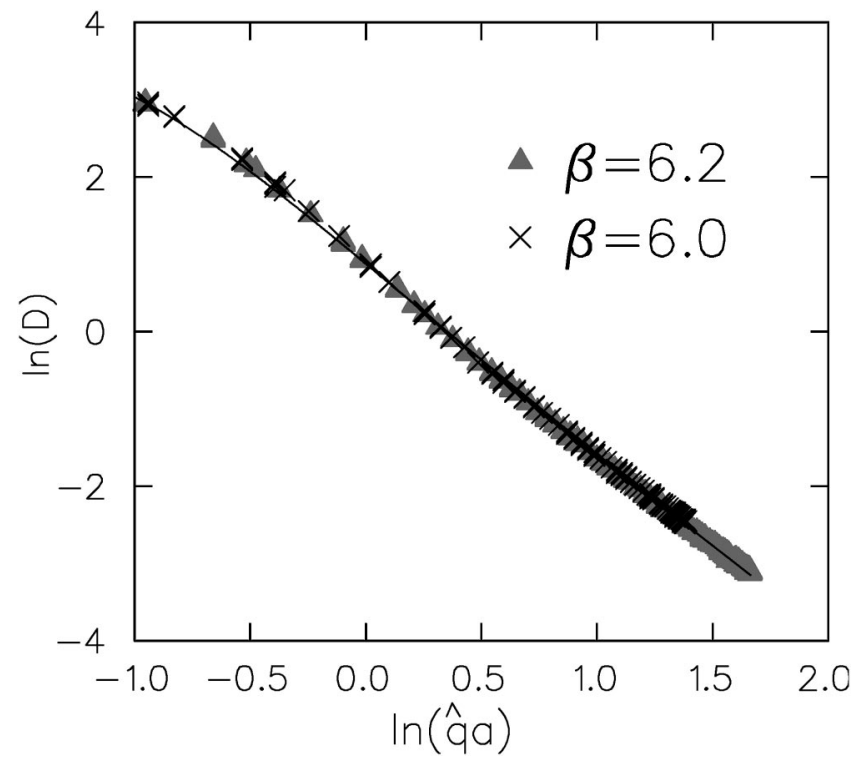

FIG. 1. Data at $\beta=6.0\left(16^{3} \times 32\right.$ lattice; crosses $)$ and at $\beta$ $=6.2\left(24^{3} \times 48\right.$ lattice; triangles $)$ fall on a universal curve.

here, such as the pole mass and the zero momentum limit of the gluon propagator, a lattice size of about $1.5 \mathrm{fm}$ suffices. Therefore for this study we use a lattice of spatial size $\sim 1.7 \mathrm{fm}$. Our observable is the transverse part, $D\left(q^{2}\right)$, of the propagator. The excellent scaling behavior which we observe for $D\left(q^{2}\right)$ enables us to extract accurately the change with $\beta$ of the lattice spacing. In the infrared, it allows us to control the cutoff effects on the pole mass and to study its continuum limit. Our notation is the same as that of Ref. [8] and we refer the reader to [8] for the details of our approach.

\section{SCALING}

Reasonable scaling was already observed in [8] where we compared data at $\beta=5.8$ and 6.0 on a lattice of size $16^{3}$ $\times 32$. Here we compare $\beta=6.0$ and $\beta=6.2$.

The results at $\beta=6.2$ were obtained from 220 configurations generated by the UKQCD Collaboration on a lattice of size $24^{3} \times 48$. At $\beta=6.0$ we used 200 configurations of size $16^{3} \times 32$ from the NERSC archive [14]. Our Laplacian gauge condition consists of aligning along a fixed orientation the local 3-color frame built from the two lowest-lying eigenvectors of the covariant Laplacian. For implementation details, see [8]. Being now closer to the continuum limit, we find very good scaling behavior for $D\left(q^{2}\right)$ as demonstrated in Fig. 1, where the two data sets fall on the same curve after applying the linear transformation

$$
\ln \left[D_{\beta=6.0}\left(\ln q a_{\beta=6.0}\right)\right]=\ln \left[D_{\beta=6.2}\left(\ln q a_{\beta=6.2}-b\right)\right]+c .
$$

The two fitted parameters take values $b=0.277 \pm 0.022$ and $c=-0.574 \pm 0.053$, which yields the scaling ratios

$$
\frac{Z_{\beta=6.2}}{Z_{\beta=6.0}}=1.02 \pm 0.14, \quad \frac{a_{\beta=6.2}}{a_{\beta=6.0}}=0.758 \pm 0.017
$$

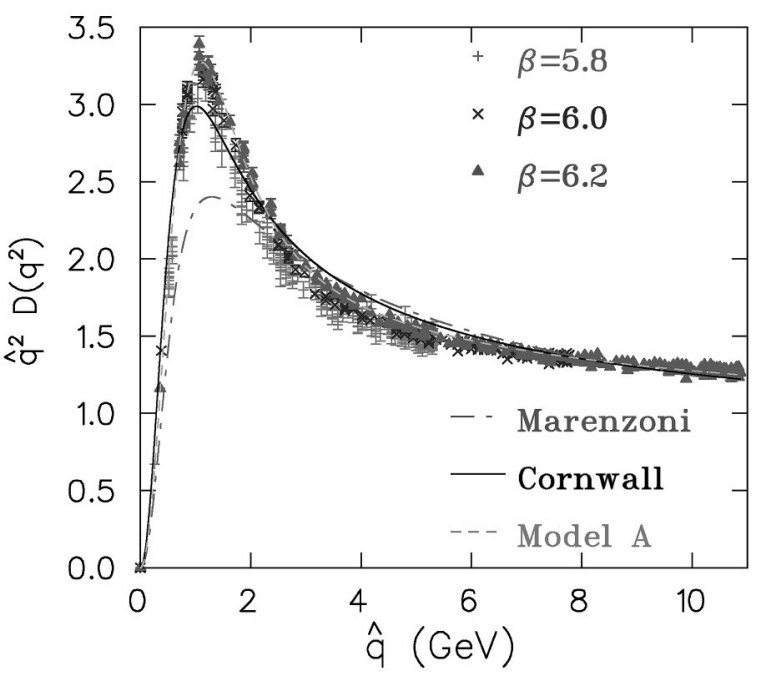

FIG. 2. The transverse gluon propagator multiplied by $\hat{q}^{2}$ at $\beta$ $=5.8,6.0$, and 6.2 in physical units. The fits to three models are shown: Marenzoni (dashed-dotted line) [17], Cornwall (solid line) [10], and model A (dashed line) of Ref. [7].

This ratio of lattice spacings is consistent with that obtained from measurements of the string tension [15], and very close to the value 0.729 obtained from the interpolation formula of the ALPHA Collaboration [16] for $r_{0} / a$. Therefore, we use for the lattice spacing the value $a^{-1}(\beta=6.2)=2.718 \mathrm{GeV}$ of Ref. [15], corresponding to a string tension $\sqrt{\sigma}$ $=440 \mathrm{MeV}$.

The renormalized zero momentum propagator also exhibits good scaling. We obtain a value of $\left[454(5) \mathrm{MeV}^{-2}\right.$ in agreement with our previous value of $\sim(445 \mathrm{MeV})^{-2}$, both for a renormalization point of $1.943 \mathrm{GeV}$.

Our new transverse propagator data at $\beta=6.2$ are shown in Fig. 2 together with our previous results. Since the $\beta$ $=6.0$ and 6.2 data agree so well over the whole momentum range, we can compare them to a variety of continuum models. We find that infrared enhancement such as $\left(q^{2}\right)^{-2}[4]$, as well as the Gribov-type infrared suppression of the form $D\left(q^{2}\right)=Z q^{2} /\left(q^{4}+M^{4}\right) L\left(q^{2}, M^{2}\right)$ [17] or $Z q^{2} /\left(q^{4}+2 a q^{2}\right.$ $\left.+M^{4}\right) L\left(q^{2}, M^{2}\right)[5]$ are both excluded by our data. Here, the function $L\left(q^{2}, M^{2}\right)$ enforces the perturbative ultraviolet behavior of the propagator:

$$
L\left(q^{2}, M^{2}\right)=\left\{\frac{1}{2} \ln \left[\left(q^{2}+M^{2}\right)\left(q^{-2}+M^{-2}\right)\right]\right\}^{-13 / 22} .
$$

The ansatz of Marenzoni et al. [18],

$$
D\left(q^{2}\right)=Z \frac{M^{2 \alpha}}{\left(q^{2}\right)^{1+\alpha}+\left(M^{2}\right)^{1+\alpha}},
$$

with a nonperturbative anomalous dimension $\alpha$, gives a better description of the lattice data $\left[\chi^{2} /\right.$ degrees of freedom $\left.(\mathrm{DOF})=18\right]$ but clearly underestimates the peak in Fig. 2. A much better description is provided by model A of Ref. [7]: 


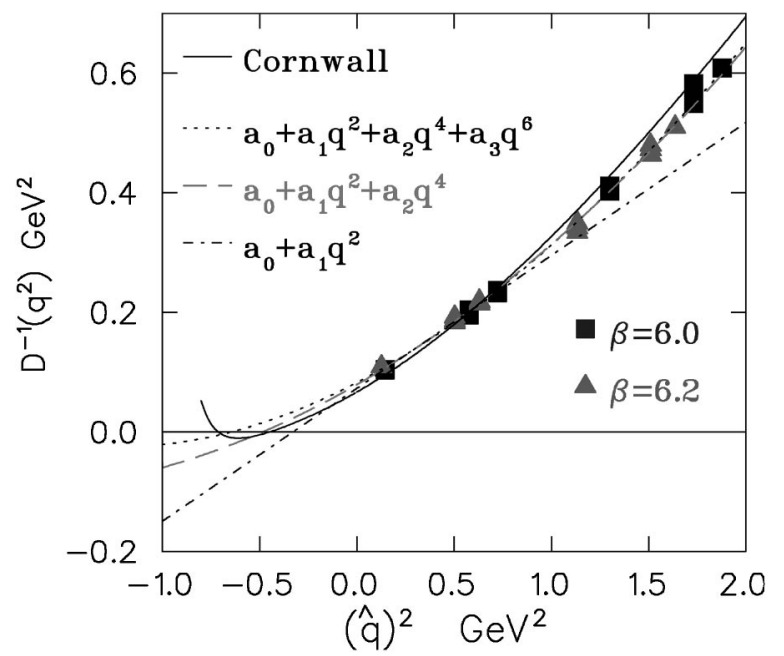

FIG. 3. Infrared inverse gluon propagator at $\beta=6.0$ (squares) and 6.2 (triangles). The various extrapolations to negative $\hat{q}^{2}$ are linear (dot-dashed), quadratic (dashed), and cubic (dotted) polynomials, and Cornwall's model (solid line).

$$
D\left(q^{2}\right)=Z\left[\frac{A M^{2 \alpha}}{\left(q^{2}+M^{2}\right)^{1+\alpha}}+\frac{1}{q^{2}+M^{2}} L\left(q^{2}, M^{2}\right)\right]
$$

with $L\left(q^{2}, M^{2}\right)$ defined in Eq. (2). Although this model gives the best fit to our data $\left(\chi^{2} / \mathrm{DOF} \sim 2\right)$, it requires one more parameter, and lacks a theoretical motivation. Worse for us here, it cannot be analytically continued.

Taking a different approach, Cornwall [10] allows for a dynamically generated gluon mass which vanishes at large momentum in accord with perturbation theory:

$$
D\left(q^{2}\right)=Z\left[\left[q^{2}+M^{2}\left(q^{2}\right)\right] \ln \frac{q^{2}+4 M^{2}\left(q^{2}\right)}{\Lambda^{2}}\right]^{-1}
$$

with

$$
M\left(q^{2}\right)=M\left\{\frac{\ln \left[\left(q^{2}+4 M^{2}\right) / \Lambda^{2}\right]}{\ln \left[4 M^{2} / \Lambda^{2}\right]}\right\}^{-6 / 11} .
$$

As we already observed on coarser lattices, we find that also at $\beta=6.2$ Cornwall's ansatz provides a reasonable fit to the data over the whole momentum range $\left(\chi^{2} / \mathrm{DOF}=7\right)$. We will thus keep it as one possible way to extrapolate to negative values of $q^{2}$ for the determination of the pole mass.

\section{POLE MASS}

As explained in the Introduction, a phenomenologically important question is whether the gluon propagator has a pole mass. The pole is a zero of the inverse propagator $D^{-1}\left(q^{2}\right)$. We show this quantity in physical units on Fig. 3, combining our data at $\beta=6.2$ and $\beta=6.0$. To determine the pole mass from $D^{-1}\left(q^{2}\right)=0$, an analytic continuation to negative $q^{2}$ is needed. Given our finite amount of data, this

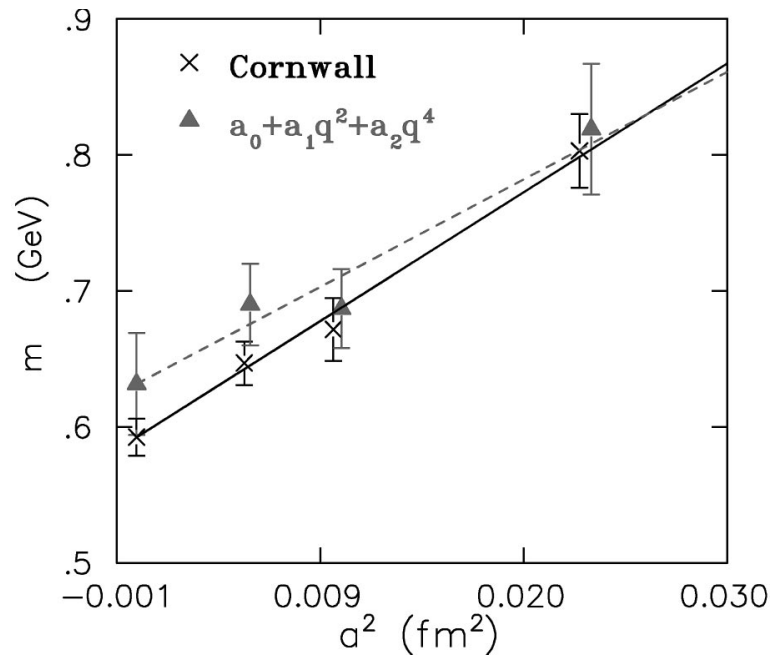

FIG. 4. The pole mass at $\beta=5.8,6.0$, and 6.2 extracted from fitting to a quadratic polynomial (data shifted to the right for clarity) and to Cornwall's ansatz, as a function of the lattice spacing $a^{2}$. The continuum values are obtained by linear extrapolation in $a^{2}$.

extrapolation procedure is ambiguous. It is essential to test the robustness of a possible pole by comparing a variety of plausible extrapolations. Fortunately, the deviation of $D^{-1}\left(q^{2}\right)$ from a linear function of $q^{2}$ in the infrared is small (much smaller than in Landau gauge [8]), which increases confidence in the extrapolation. We consider here linear, quadratic, and cubic polynomials in $q^{2}$, as well as Cornwall's ansatz (5). All four choices indicate the presence of a pole. The variation in its location gives us crude systematic error estimates.

Given the curvature of the data, a linear fit provides a lower bound for the pole mass, with a value of $505 \mathrm{MeV}$. This mass increases to 693(20) MeV with a quadratic fit, and reaches $800 \mathrm{MeV}$ for a cubic fit. Note that the fitted coefficients of the higher powers of $q^{2}$ keep decreasing, which indicates the soundness of an extrapolation based on a Taylor expansion. However our data are not sufficient to reliably fit higher-degree polynomials: in particular, the results depend on the fitted momentum interval and on the presence or absence of data removed by the "cylindrical cut" of Ref. [7], whose purpose is to eliminate momenta most affected by lattice artifacts. In comparison with these simple polynomial fits, Cornwall's ansatz gives a pole mass of 669(6) MeV, consistent with that of the quadratic polynomial. The various extrapolations are shown together in Fig. 3.

By performing the same analysis at the three values of the lattice spacing $a(\beta), \beta=5.8,6.0$, and 6.2 we have studied, we can extrapolate the pole mass to the continuum limit. In Fig. 4 we compare such an extrapolation in $a^{2}$ for pole masses obtained by fitting a quadratic polynomial or Cornwall's ansatz. It can be seen that both give consistent results, at fixed lattice spacing as well as in the continuum. The continuum values are 632(38) MeV and 592(14) MeV using the quadratic polynomial and Cornwall's ansatz, respectively.

The reasonable robustness of our analysis, with respect to a change in the lattice spacing as well as in the analytic 


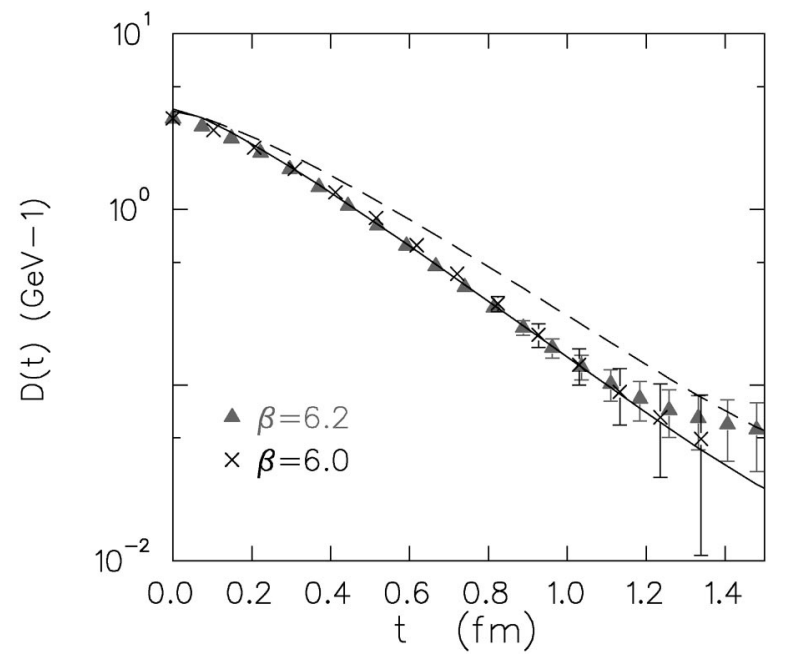

FIG. 5. Time-slice gluon correlator at $\beta=6.0$ and 6.2. The dashed line shows Cornwall's model fitted to $D^{-1}\left(\hat{q}^{2}\right)$ after the cylindrical momentum cut; the solid line is a direct fit of the same model to the time-slice correlators, excluding the first few time slices.

continuation procedure, leads us to conclude that there is strong evidence that a pole exists in the gluon propagator, with a mass of about $600 \mathrm{MeV}$. Because of the curvature of the inverse propagator, the systematic error in the extrapolation to negative $q^{2}$ is asymmetric. From the variation observed above with the choice of analytic continuation, we estimate it at $\sim-30,+150 \mathrm{MeV}$. Based on our study of finite volume effects [8], and given our present lattice size, we expect negligible finite size corrections to this pole mass. Comparing it to the glueball mass of $1.73(0.05)(0.08) \mathrm{GeV}$ [19], it appears close to one-third of the glueball mass (rather than one-half as sometimes speculated). A pole mass of $500-800 \mathrm{MeV}$ is also within the range needed to fit experimental data in various phenomenological studies $[11,12]$.

As a further, model-independent check on the value of the pole mass, we measured the correlator of the gluon field averaged over a time slice: namely [8],

$$
C(t)=\frac{1}{L_{s}^{3}} \frac{1}{8} \sum_{a=1}^{8} \frac{1}{3} \sum_{\mu=1}^{3}\left(\sum_{x}^{L_{s}^{3}} A_{\mu}^{a}(\vec{x}, 0)\right)\left(\sum_{x}^{L_{s}^{3}} A_{\mu}^{a}(\vec{x}, t)\right)
$$

The exponential decay, if at all, of this correlator at large time is governed by the pole mass. Therefore, one can in principle measure the pole mass in a model-independent way. However, statistical noise limits the usefulness of this approach. We display the correlator in Fig. 5. The data are of insufficient quality to measure an exponential fall off, and a fitting procedure is needed. Although this correlator is measured on the same configurations as $D\left(q^{2}\right)$, the various momenta are given a different weight, so that a fit to $C(t)$ will give different results than a fit to $D^{-1}\left(q^{2}\right)$, especially after the cylindrical momentum cut in the latter. Since all momenta enter in $C(t)$, we fit the only ansatz which describes the gluon propagator reasonably well over the whole momentum range, namely Cornwall's model, directly to $C(t)$ instead of $D^{-1}\left(q^{2}\right)$ as before. The dashed line in Fig. 5 shows the original fit of Cornwall's ansatz to $D^{-1}\left(q^{2}\right)$, which already provides a fair description of the data. The solid line represents a direct fit of the same 3-parameter ansatz to $C(t)$, excluding the first few time slices which are contaminated by contributions from excited states. A simultaneous fit of the time-slice correlator data at $\beta=6.2$ and 6.0 yields a pole mass of $739(81) \mathrm{MeV}$, in agreement with the value $669(6) \mathrm{MeV}$ extracted from $D^{-1}\left(q^{2}\right)$, but with much larger statistical errors.

If one attempts a model-independent determination of the pole mass from the effective mass $m_{\mathrm{eff}}(t)=-\operatorname{Ln}[C(t$ $+1) / C(t)]$, one obtains a value of 702(163) MeV, poorly determined but consistent with the direct fit. Therefore, our different analyses give pole masses ranging from $592 \mathrm{MeV}$ (continuum extrapolation of pole of propagator fitted by Cornwall's ansatz) to $739 \mathrm{MeV}$ (fit of time-slice correlator). Taking into account the asymmetry of potential systematic errors, we estimate the gluon pole mass to be $\sim 600_{-30}^{+150} \mathrm{MeV}$.

\section{CONCLUSIONS}

We have extended a previous lattice study of the gluon propagator in the Laplacian gauge to a finer lattice and found good scaling behavior. We confirm the existence of a pole as we approach the continuum limit. Applying a variety of different fits we extract a pole mass in the range of 600-30 $+150 \mathrm{MeV}$ in accord with the value found in phenomenological studies for the description of hadron-hadron scattering.

It would be very interesting to substantiate the gauge invariance of the gluon propagator pole mass by similar studies in other gauges. Reference [20] proposes a nonlocal, gaugeinvariant gluon propagator based on the (Coulomb-like) Laplacian gauge in three dimensions. It is argued there that the pole mass of this propagator determines the vectorpseudoscalar-mass splitting $M_{V}-M_{S}$ in heavy quarkonia. This relation is consistent with the $S U(2)$ numerical results presented. For QCD, using the experimental values for the splitting in $c \bar{c}$ and $b \bar{b}$ systems, the implication is that the pole mass is $\sim 420 \mathrm{MeV}$. This is somewhat low compared to our estimate. In Landau gauge, our first attempt [8] showed no indication for a gluon propagator pole. However, lattice Gribov copies might play a crucial role there. Moreover, Landau gauge appears more sensitive than Laplacian gauge to finite-size effects. Thus this question requires larger lattices as studied in [7].

\section{ACKNOWLEDGMENTS}

The $16^{3} \times 32$ lattice configurations came from the Gauge Connection archive [14], and the $24^{3} \times 48$ were provided by the UKQCD Collaboration. 
[1] C. D. Roberts and S. M. Schmidt, Prog. Part. Nucl. Phys. 45S1, 1 (2000).

[2] Z. Fodor and S. D. Katz, J. High Energy Phys. 03, 014 (2002); Nucl. Phys. B (Proc. Suppl.) 106, 441 (2002); QCD-TARO Collaboration, S. Choe et al., Nucl. Phys. A698, 395 (2002); Nucl. Phys. B (Proc. Suppl.) 106, 462 (2002); S. Ejiri et al., ibid. 106, 459 (2002).

[3] G. B. West, Phys. Rev. D 27, 1878 (1983).

[4] S. Mandelstam, Phys. Rev. D 20, 3223 (1979); N. Brown and M. R. Pennington, ibid. 38, 2266 (1988); 39, 2723 (1989).

[5] U. Habel, R. Könning, H. G. Reusch, M. Stingl, and S. Wigard, Z. Phys. A 336, 423 (1990); 336, 435 (1990); M. Stingl, ibid. 353, 423 (1996).

[6] L. von Smekal, A. Hauck, and R. Alkofer, Phys. Rev. Lett. 79, 3591 (1997); Ann. Phys. (N.Y.) 267, 1 (1998).

[7] D. Leinweber, J. I. Skullerud, A. G. Williams, and C. Parrinello, Phys. Rev. D 60, 094507 (1999); 61, 079901(E) (2000); F. D. R. Bonnet, P. O. Bowman, D. B. Leinweber, and A. G. Williams, ibid. 62, 051501(R) (2000).

[8] C. Alexandrou, Ph. de Forcrand, and E. Follana, hep-lat/0009003; Phys. Rev. D 63, 094504 (2001).

[9] R. Kobes, G. Kunstatter, and A. Rebhan, Phys. Rev. Lett. 64, 2992 (1990); Nucl. Phys. B355, 1 (1991).

[10] J. M. Cornwall, Phys. Rev. D 26, 1453 (1982).

[11] G. Parisi and R. Petronzio, Phys. Lett. 94B, 51 (1980).

[12] F. Halzen, G. Klein, and A. A. Natale, Phys. Rev. D 47, 295 (1993); M. B. Gay Ducati, F. Halzen, and A. A. Natale, ibid. 48, 2324 (1993).

[13] P. V. Landshoff and O. Nachtmann, Z. Phys. C 35, 405 (1987).

[14] http://qcd.nersc.gov.

[15] G. S. Bali and K. Schilling, Phys. Rev. D 47, 661 (1993).

[16] ALPHA Collaboration, M. Guagnelli, R. Sommer, and H. Wittig, Nucl. Phys. B535, 389 (1998).

[17] V. N. Gribov, Nucl. Phys. B139, 1 (1978).

[18] P. Marenzoni, G. Martinelli, N. Stella, and M. Testa, Phys. Lett. B 318, 511 (1993); Nucl. Phys. B455, 339 (1995).

[19] C. Morningstar and M. Peardon, Phys. Rev. D 60, 034509 (1999).

[20] O. Philipsen, Phys. Lett. B 521, 273 (2001); Nucl. Phys. B (Proc. Suppl.) 106, 242 (2002). 